\title{
Prophylactic Application of Lavandula Angustifolia Mill. Oil and Its Active Component Linalyl Acetate Alleviate Comorbidity and Exacerbation Risk in Rats With Collagen-Induced Arthritis Accompanied by Chronic Nicotine-Induced Hypertension.
}

\author{
Eunhye Seo \\ Korea University \\ Yu Shan Hsieh \\ Korea University \\ Jeong-Min Lee \\ KT\&G Central Research Institute \\ You Kyoung Shin \\ Korea University \\ Geun Hee Seol ( $\nabla$ ghseol@korea.ac.kr) \\ Korea University https://orcid.org/0000-0002-2986-9688
}

\section{Research}

Keywords: Lavandula angustifolia Mill, Linalyl acetate, Rheumatoid arthritis, Hypertension

Posted Date: December 16th, 2020

DOI: https://doi.org/10.21203/rs.3.rs-127467/v1

License: (a) (1) This work is licensed under a Creative Commons Attribution 4.0 International License. Read Full License 


\section{Abstract}

Background: Hypertension (HTN) in rheumatoid arthritis (RA) is more common in smokers than in nonsmokers, increasing the risks of cardiovascular and skeletal muscle related comorbidities, but studies on pharmacological protection strategies are insufficient. Lavandula angustifolia Mill. (lavender) oil has traditionally been used for RA and HTN via its anti-inflammatory properties. This study aimed to investigate the effects of lavender oil and its main component linalyl acetate on cardiovascular and skeletal muscle protection in a rat model of RA with chronic nicotine-induced HTN.

Methods: Collagen-induced arthritis (CIA), with or without chronic nicotine-induced $\mathrm{HTN}$, were induced in Sprague-Dawley rats. Lavender or linalyl acetate (10 or $100 \mathrm{mg} / \mathrm{kg} /$ day) were administrated intraperitoneally to CIA plus chronic nicotine-induced HTN (H-CIA) rats. The effects of lavender and linalyl acetate was evaluated by measurement of hind paw thickness, body weight, blood pressure (BP), serum concentrations of interleukin-6 (IL-6) and insulin-like growth factor-1 (IGF-1). In addition to above, histological analysis of thoracic aorta and gastrocnemius muscle were used.

Results: BP was significantly higher, vascular endothelium more deteriorated and paw edema more severe in $\mathrm{H}-\mathrm{CIA}$ than in CIA. Both groups showed clear signs of skeletal muscle atrophy and inflammation, but gastrocnemius muscle weight was lower and muscle inflammatory status worse in H-CIA than in CIA. Reduced mitochondrial function was observed only in H-CIA. Prophylactic application of $100 \mathrm{mg} / \mathrm{kg}$ lavender oil or linalyl acetate kept IL- 6 levels to the normal range and reduced hind paw edema and systolic BP. Linalyl acetate $100 \mathrm{mg} / \mathrm{kg}$ was the only treatment that maintained diastolic BP and IGF-1 in their normal ranges and prevented vascular endothelial damage. These treatments also reduced inflammation of the gastrocnemius muscle and inhibited mitochondrial dysfunction while tending to increase muscle mass.

Conclusions: Chronic nicotine exposure induces defects that increase the risks of cardiovascular disease and muscle weakness, and application of lavender and linalyl acetate reduced the risks, especially, linalyl acetated alleviated vascular endothelial damage along with maintained IGF-1 level. Current results encourage smokers at high risk for RA to quit smoking, and further suggest that linalyl acetate is a useful agent to prevent cardiovascular and skeletal muscle related comorbidities.

\section{Background}

Although the development of effective drugs and regular adjustment of treatment have improved the prognosis of patients with rheumatoid arthritis (RA), comorbidities can reduce their survival and quality of life [1]. These negative outcomes may be due the increased prevalence of cardiovascular diseases and functional impairments in RA patients, which may be exacerbated by hypertension (HTN) [2]. HTN is more frequent in patients with RA than in the general population, with $62 \%$ of men and $47 \%$ of women with early RA having HTN [3]. HTN also increases the risk of cardiovascular disease, which has been a primary or underlying cause of death in $52.6 \%$ of RA patients [4]. Moreover, muscle weakness which is common in 
patients with RA, is likely to be exacerbated by HTN [2]. Despite these associations, blood pressure (BP) is not effectively controlled in RA patients, for example, $50 \%$ of RA with HTN patients maintained SBP above $140 \mathrm{mmHg}$ even when taking antihypertensive drugs [5]. Therefore, there is a need to develop agents to prevent cardiovascular and skeletal muscle related comorbidities in RA with HTN patients, but there is currently no definitive way.

Tobacco smoking is representative behavioral risk factor for the development of HTN [6], and cumulative exposure to nicotine has a substantial negative impact on outcomes in patients with RA [7]. Little is known about the management of comorbidities in patients with RA, including cardiovascular and skeletal muscle related diseases, especially in smokers. In this context, we have developed a rat model of RA with HTN, in which rats are subjected to chronic exposure to nicotine and injection of collagen. We have used a combination model of RA and HTN to test the ability of herbal extracts to protect rats from cardiovascular and skeletal muscle related diseases.

Lavandula angustifolia Mill. (lavender) is an aromatic shrub which grows worldwide, and its oil is used for making cosmetics, perfumes, soaps, detergents and food flavors [8]. Lavender oil has been widely used for its analgesic [9] and anti-inflammatory [10,11] effects in chronic disease. For example, lavender oil was one of the most commonly used herbal medications in combination with standard treatment by RA patients [12], and massage with lavender oil significantly reduced the pain severity in patients with knee osteoarthritis [13]. In addition, lavender oil has possibility of preventing cardiovascular disease based on anti-diabetic [14] and antithrombotic activities [15]. Linalyl acetate, a volatile and low toxicity compound contained in citrus fruits, is the main component of lavender oil [16] Linalyl acetate has shown anti-inflammatory effects in vitro [17] and in vivo [18], as well as anti-hypertensive [19], anti-diabetic [20] and anti-stress [21] effects with the ability to prevent vascular endothelium dysfunction [18]. These pharmacological properties suggested that lavender and linalyl acetate could have preventive effects in our rat model of RA with chronic nicotine-induced HTN. The present study was therefore performed to examine the potential preventive effects of lavender oil and linalyl acetate in our rat model of RA with chronic nicotine-induced HTN.

\section{Materials And Methods}

\section{Animals and chemicals}

Male Sprague-Dawley rats aged 7 weeks were purchased from Youngbio (Seongnam, Korea) and maintained $2 \sim 4$ per cage at $20 \sim 25^{\circ} \mathrm{C}$ with a 12-h light-dark cycle. Rat chow and tab water were allowed ad libitum. All procedures were approved by the institutional animal care and research committee of Korea University (KUIACUC-2016-153). Nicotine hydrogen tartrate salt, type II collagen, complete and incomplete Freund's adjuvant, polyethylene glycol and linalyl acetate were obtained from Sigma-Aldrich (St Louis, MO, USA). Lavender oil was purchased from Aromarant Co. Ltd. (Rottingen, Germany).

Gas chromatography/mass spectrometry profiling of Lavandula angustifolia Mill. oil. 
Gas chromatography/mass spectroscopy (GC/MS) profiling of the non-polar constituents in Lavandula angustifolia Mill. oil (Aromarant Co. Ltd., batch no. 180622, Rottingen, Germany) was done using a GC/MS (8890-5977B GC-MS, Agilent, USA). The extract was dissolved and transferred to GC- vials and run on the GC/MS. The instrument was blank with Supleco methanol. The chromatographic conditions were as follows: the samples were separated on a HP-5MS capillary columng (30 $\mathrm{m} \times 0.25 \mathrm{~mm} \times$ $0.25 \mu \mathrm{m}$, Agilent J\&W Scientific, USA), and the injection volume was $1 \mu \mathrm{L}$ with a spilt ratio 100:1. The inlet temperature was $230^{\circ} \mathrm{C}$, highly pure helium gas was used as the carrier gas, and the flow rate was 1.2 $\mathrm{mL} / \mathrm{min}$. The initial temperature was $40^{\circ} \mathrm{C}$, which was maintained for $10 \mathrm{~min}$, and the temperature was increased to $300^{\circ} \mathrm{C}$ at the rate of $5^{\circ} \mathrm{C} / \mathrm{min}$. The final temperature was held for $10 \mathrm{~min}$. The mass spectrum conditions were as follows: ion source temperature of $230^{\circ} \mathrm{C}$, transfer line temperature of $250^{\circ} \mathrm{C}$, full scanning mode, mass scanning range of $\mathrm{m} / \mathrm{z} 40-500$. Identification of the non-polar phytoconstituents was by comparison with those available in the National Institute of Standards and Technology (NIST) library coupled to the instrument.

\section{Experimental protocol}

Fifty-two rats were randomly allocated to seven groups: a normal control (NC) group, a collagen-induced arthritis (CIA) group, a CIA plus chronic nicotine-induced HTN (H-CIA) group, and H-CIA groups treated with $10 \mathrm{mg} / \mathrm{kg}$ lavender, $100 \mathrm{mg} / \mathrm{kg}$ lavender, $10 \mathrm{mg} / \mathrm{kg}$ linalyl acetate, and $100 \mathrm{mg} / \mathrm{kg}$ linalyl acetate. CIA and chronic nicotine-induced HTN were induced simultaneously (Fig. 2). HTN was induced by intraperitoneal injection of $0.8 \mathrm{mg} / \mathrm{kg} /$ day nicotine for 3 weeks, starting on the first experimental day, followed by a boost of $3 \mathrm{mg} / \mathrm{kg}$ nicotine on day 22 [22]. CIA was induced by injection of $2 \mathrm{mg} / \mathrm{ml}$ type II collagen obtained from chicken sternal cartilage and dissolved in $0.01 \mathrm{~N}$ glacial acetic acid emulsified in an equal volume of complete Freund's adjuvant, and a second injection of type II collagen emulsified in incomplete Freund's adjuvant on day 19 [23].

Lavender and linalyl acetate were dissolved in polyethylene glycol and injected intraperitoneally twice per week during the entire follow up period. All groups of rats were followed-up for 35 days. Hind paw thickness, body weight, and BP were measured on days $0,8,15,22,29$, and 35 . At the end of the experimental protocol, blood was obtained from the inferior vena cava and the serum separated by centrifugation at $3,000 \mathrm{~g}$ for $20 \mathrm{~min}$. The thoracic aorta and both gastrocnemius muscles were carefully removed from each rat for further evaluation.

\section{Hind paw thickness and BP measurements}

Joint edema was assessed as the average thickness on both sides of hind paw that measured using calipers (Mitutoyo, Japan), as previously described [24]. BP was measured using a volume-pressure recording tail cuff method (CODA-6 System, Kent Scientific, Torrington, CT). Rats were placed in a holding device maintained at $38^{\circ} \mathrm{C}$ on a heating plate for 10 minutes. BP was measured 15 times, and the average value were used.

\section{Measurements of aorta endothelium thickness}


Extracted aortae were fixed in 4\% paraformaldehyde and sectioned into 4 um-thick slices. The slices were stained with hematoxylin and eosin (H\&E) and imaged at 400x magnification with a Nikon DS-Ri2 fluorescence microscope (Nikon, Japan). Images were captured with a digital camera attached to the microscope, and endothelium thickness per $50 \mu \mathrm{m}$ was determined by NIS Elements imaging software (Nikon, Japan), using the formula: total vessel wall diameter - (outer vessel wall diameter + middle vessel wall diameter). Arterial endothelium thickness was calculated as the average of three measurements along the wall of each artery.

\section{Measurements of serum interleukin-6 (IL-6) and insulin-like growth factor-1 (IGF-1) concentrations}

Concentrations of serum IL- 6 and IGF-1 were measured using rat IL-6 (Komabiotech, Korea) and IGF-1 (Abcam, UK) Enzyme-Linked Immunosorbent Assay (ELISA) kits, respectively. Results were obtained by measuring absorbance at $405 \mathrm{~nm}$ using a microplate ELISA reader (BMG Labtech, Germany), according to the manufacturer's instructions.

\section{Histopathological analysis of muscle tissue}

The inflammatory status of gastrocnemius muscle tissue was assessed histopathologically. Muscle tissues fixed in $4 \%$ paraformaldehyde and embedded to paraffin blocks were cut into $4-\mu m$-thick sections, which were mounted onto slides. The slides were stained with H\&E and imaged at 400x magnification using a Nikon DS-Ri2 fluorescence microscope (Nikon, Japan). The threshold of each of the three images was measured the background, connective tissue, and total tissue by Image $\mathrm{J}$ software (National Institutes of Health, USA). Percentage of fibrosis in the tissue was calculated as follows: background area was subtracted from connective tissue and total tissue, then followed the formula: percentage of fibrosis $=$ connective tissue $/$ connective tissue + total tissue $\times 100$ [25].

\section{Measurement of mitochondrial membrane potential}

Muscle tissue samples were homogenized and lysed for $30 \mathrm{~min}$ on ice with lysis buffer and centrifuged for $15 \mathrm{~min}$ at $13,000 \mathrm{~g}$. Mitochondrial membrane potential was determined using a fluorescent JC1Mitochondrial Membrane Potential Assay Kit (Abcam, UK). The fluorescence intensities of the aggregate and monomer forms were measured with a fluorescence microplate reader (PerkinElmer, Korea) operated at excitation/emission wavelengths of 535 and $485 \mathrm{~nm}$, respectively. The results are presented as the ratio of aggregate/monomer fluorescence intensity.

\section{Statistics}

All data are presented as means \pm SEM, with percentages reported relative to the $\mathrm{H}$-CIA group. Multiple groups were compared by one-way ANOVA followed by Tukey's honestly significant difference (HSD) post hoc tests. All statistical analyses were performed using SPSS version 24.0 software (SPSS, Chicago, IL), with $p<0.05$ considered statistically significant. 


\section{Results}

\section{GC/MS profiling of Lavandula angustifolia Mill. oil}

The main abundant of these compounds are Linalool and Linalyl acetate which constitute about $14.46 \%$, $13.68 \%$, respectively, of the volatile constituents of the extract. Other compounds with relative abundance include cis-Caryophyllene (4.57\%), Terpinen-4-ol (4.37\%), trans-Caryophyllene (4.23\%), $\beta$-Ocimene (3.67\%), Isoborneol (3.61\%), a-Ocimene (3.30\%), 5-Methyl-2-prop-1-en-2-ylhex-4-en-1-ol (3.27\%) (Table 1). 
Table 1

Chemical profile identified by GC/MS analysis of Lavandula angustifolia Mill. oil.

\begin{tabular}{|c|c|c|c|}
\hline No & RT & Compound & Area \% \\
\hline 1 & 6.413 & 1-Methoxyhexane & 0.92 \\
\hline 2 & 8.030 & (E)-3-Hexen-1-ol & 0.06 \\
\hline 3 & 8.971 & 1-Hexanol & 0.26 \\
\hline 4 & 11.992 & Butyl propionate & 0.03 \\
\hline 5 & 12.204 & Tricyclene & 0.10 \\
\hline 6 & 12.708 & a-Phellandrene & 0.50 \\
\hline 7 & 12.989 & a-Pinene & 0.85 \\
\hline 8 & 13.322 & 3,5-Dimethylphenol & 0.02 \\
\hline 9 & 13.762 & Camphene & 0.51 \\
\hline 10 & 14.549 & Butyl isobutyrate & 0.04 \\
\hline 11 & 15.032 & o-Cymene & 0.03 \\
\hline 12 & 15.260 & $\beta$-Pinene & 0.30 \\
\hline 13 & 15.787 & 1-Octen-3-ol & 1.17 \\
\hline 14 & 16.097 & 3-Octanone & 2.05 \\
\hline 15 & 16.263 & $\beta$-Myrcene & 1.50 \\
\hline 16 & 16.524 & 3-Octanol & 0.81 \\
\hline 17 & 16.590 & Butyl butanoate & 0.68 \\
\hline 18 & 16.922 & (+)-3-Carene & 0.32 \\
\hline 19 & 17.246 & a-Terpinene & 0.06 \\
\hline 20 & 17.378 & Hexyl acetate & 1.79 \\
\hline 21 & 17.510 & m-Cymene & 0.42 \\
\hline 22 & 17.598 & p-Cymene & 1.81 \\
\hline 23 & 17.754 & D-Limonene & 0.59 \\
\hline 24 & 17.822 & Eucalyptol & 0.55 \\
\hline 25 & 18.339 & $\beta$-Ocimene & 3.67 \\
\hline 26 & 18.726 & a-Ocimene & 3.30 \\
\hline 27 & 19.027 & $\mathrm{Y}$-Terpinene & 0.27 \\
\hline
\end{tabular}




\begin{tabular}{|c|c|c|c|}
\hline No & RT & Compound & Area \% \\
\hline 28 & 19.346 & 5-Isopropyl-2-methylbicyclo[3.1.0]hexan-2-ol & 0.08 \\
\hline 29 & 20.129 & a-Terpinolene & 0.18 \\
\hline 30 & 20.554 & 3-Methyl-2-(2-methyl-2-butenyl)-furan & 0.18 \\
\hline 31 & 20.735 & Linalool & 14.46 \\
\hline 32 & 21.215 & 1-Octen-3-yl-acetate & 2.53 \\
\hline 33 & 21.592 & 3-Octyl acetate & 0.93 \\
\hline 34 & 22.095 & $(+)$-2-Bornanone & 1.27 \\
\hline 35 & 22.380 & Hexyl isobutyrate & 0.36 \\
\hline 36 & 22.825 & Isoborneol & 3.61 \\
\hline 37 & 22.991 & 5-Methyl-2-prop-1-en-2-ylhex-4-en-1-ol & 3.27 \\
\hline 38 & 23.246 & Terpinen-4-ol & 4.37 \\
\hline 39 & 23.492 & 4-Isopropyl-2-cyclohexenone & 0.69 \\
\hline 40 & 23.663 & a-Terpineol & 2.66 \\
\hline 41 & 23.762 & Hexyl butanoate & 1.81 \\
\hline 42 & 23.901 & (E)-2,6-Dimethylocta-3,7-diene-2,6-diol & 0.81 \\
\hline 43 & 24.926 & Nerol & 1.02 \\
\hline 44 & 25.646 & Linalyl acetate & 13.68 \\
\hline 45 & 26.560 & Bornyl acetate & 0.70 \\
\hline 46 & 27.755 & Hexyl tiglate & 0.84 \\
\hline 47 & 28.672 & Neryl acetate & 1.87 \\
\hline 48 & 28.975 & a-Cubebene & 0.15 \\
\hline 49 & 29.196 & Geranyl acetate & 2.67 \\
\hline 50 & 29.361 & 1,3-Cyclohexadiene, 5-(1,5-dimethyl-4-hexenyl)-2-methyl- & 0.74 \\
\hline 51 & 30.186 & trans-Caryophyllene & 4.23 \\
\hline 52 & 30.524 & trans-a-Bergamotene & 1.27 \\
\hline 53 & 30.652 & iso-Amyl tiglate & 0.15 \\
\hline 54 & 30.991 & Humulene & 1.00 \\
\hline 55 & 31.075 & cis-Caryophyllene & 4.57 \\
\hline
\end{tabular}




\begin{tabular}{|llll|}
\hline No & RT & Compound & Area \% \\
\hline 56 & 31.697 & (+)-Cyclosativene & 2.70 \\
\hline 57 & 32.329 & $\beta$-Bisabolene & 0.39 \\
\hline 58 & 32.483 & Y-Candinene & 1.05 \\
\hline 59 & 34.159 & Caryophyllene oxide & 1.54 \\
\hline 60 & 35.436 & T-Cadinol & 1.06 \\
\hline 61 & 36.368 & a-Bisabolol & 0.18 \\
\hline 62 & 37.208 & 7-Methoxy-2H-1-benzopyran-2-one & 0.17 \\
\hline 63 & 37.533 & Nerolidyl acetate & 0.06 \\
\hline 64 & 38.096 & Benzyl Benzoate & 0.10 \\
\hline 65 & 39.726 & 6,10,14-Trimethylpentadecan-2-one & 0.04 \\
\hline
\end{tabular}

\section{Effects Of Co-occurrence Of Chronic Nicotine-induced Htn And Cia}

Typical changes in hind paw thickness and body weight were observed beginning 29 days after initial exposure to collagen and/or nicotine (Fig. 3a-b), with paw edema being especially more severe in the $\mathrm{H}$ $\mathrm{CIA}$ than in the CIA group on day 35 (Fig. 3a). BP was elevated only in the H-ClA group, suggesting that HTN occurred only in this group of rats (Fig. 3c-d). Elevated BP was consistent with the decrease in thoracic aorta endothelium thickness observed only in this group (Fig. 3e). Serum IL-6 concentration, an indicator of systemic inflammation, was significantly higher (Fig. 3f), and serum IGF-1 concentration significantly lower (Fig. 3g), in the H-CIA and CIA groups than in the NC group.

Assessment of the gastrocnemius muscles showed that localized inflammation was more severe in the $\mathrm{H}-\mathrm{ClA}$ than in the $\mathrm{NC}$ and $\mathrm{CIA}$ groups (Fig. 3h). The relative weight of the gastrocnemius muscles was significantly lower in the H-CIA and CIA groups than in the NC group, and was lower in the H-CIA than in the CIA group, indicating muscle atrophy in the $\mathrm{H}$-CIA group (Fig. 3i). The ratio of fiber cross-sectional area was also lower in the $\mathrm{H}-\mathrm{ClA}$ and $\mathrm{ClA}$ groups than in the NC group, but there was no difference between the $\mathrm{H}-\mathrm{ClA}$ and $\mathrm{CIA}$ groups (Fig. 3j). Functional muscle status seemed to be unaffected in the $\mathrm{CIA}$ group, as shown by mitochondrial membrane potential, although it was slightly higher than in the NC group (Fig. 3k). In contrast, mitochondrial membrane potential was significantly higher in the H-ClA than in the NC group (Fig. 3k), indicating that mitochondrial dysfunction caused by mitochondrial toxicity occurred only in the H-CIA group. 


\section{Effects of prophylactic application of lavender and linalyl acetate on cardiovascular and skeletal muscle profiles in rats with $\mathrm{H}-\mathrm{ClA}$}

The increase in hind paw thickness was suppressed by treatment with $100 \mathrm{mg} / \mathrm{kg}$ lavender or linalyl acetate on day 35 (Fig. 4a), and body weights were significantly higher in H-ClA rats treated with lavender or linalyl acetate than in the H-CIA group on day 35 (Fig. 4b). In addition, $100 \mathrm{mg} / \mathrm{kg}$ lavender or linalyl acetate inhibited the increases in systolic BP in the H-CIA. Linalyl acetate $100 \mathrm{mg} / \mathrm{kg}$ was the only agent that simultaneously suppressed the increases in both systolic $(124.26 \pm 1.33 \mathrm{mmHg}$ vs $119.18 \pm$ $3.90 \mathrm{mmHg}$ ) and diastolic $79.63 \pm 3.45 \mathrm{mmHg}$ vs $80.65 \pm 2.85 \mathrm{mmHg}$ ) BP to normotensive levels (Fig. 4c). Moreover, only $100 \mathrm{mg} / \mathrm{kg}$ linalyl acetate preserved endothelium thickness in the normal range, showing a mean thickness was similar with NC group $(3.67 \pm 0.12 \mu \mathrm{m}$ vs $3.61 \pm 0.17 \mu \mathrm{m})$ (Fig. $4 \mathrm{~d})$. At concentrations of $100 \mathrm{mg} / \mathrm{kg}$, both lavender and linalyl acetate effectively reduced serum IL-6 levels (Fig. 5a), with $100 \mathrm{mg} / \mathrm{kg}$ linalyl acetate reducing IL-6 level to the normal range shown by the NC group $(86.18 \pm 4.13 \mathrm{pg} / \mathrm{ml}$ vs $84.72 \pm 11.01 \mathrm{pg} / \mathrm{ml})$. In addition, the level of IGF- 1 in rats treated with $100 \mathrm{mg} / \mathrm{kg}$ linalyl acetate was comparable to that in the NC group $(2051.04 \pm 141.78 \mathrm{pg} / \mathrm{ml}$ vs $1889.30 \pm$ $114.38 \mathrm{pg} / \mathrm{ml}$ ) (Fig. 5b).

Morphological analysis of H\&E stained images of gastrocnemius muscle confirmed that lavender and linalyl acetate improved localized muscle inflammatory status (Fig. $5 \mathrm{c}$ ). Although no differences were observed in relative muscle weight and muscle fiber size between the $\mathrm{H}-\mathrm{ClA}$ and treated groups, comparative histological assessment suggested muscle enhancement in rats treated with lavender and linalyl acetate (Fig. 5d-e). Mitochondrial membrane potential in muscle tissue was significantly suppressed by lavender and linalyl acetate, verifying that all treatments could maintain mitochondrial homeostasis (Fig. 5f).

\section{Discussion}

To our knowledge, the present study is the first to develop a specific method for preventing cardiovascular and skeletal muscle related comorbidities in a rat model of RA with chronic nicotine-induced HTN.

We found that chronic exposure to nicotine was associated with deteriorated cardiovascular profiles in rats at high risk of RA. That is, cumulative smoking behavior and risk factors for RA synergistically induced a rapid elevation of BP and acceleration of atherosclerosis, increasing the risks of cardiovascular events beginning at early stage of RA [26]. The reduced vascular endothelial thickness observed in the current study suggests that the endothelium is more susceptible to apoptosis, resulting in the subsequent development of atherosclerotic plaques [27]. Although vascular endothelial damage has been previously reported in RA [28], vascular damage in the present study was observed only in rats with chronic nicotineinduced HTN plus RA, a condition mimicking mild RA [23]. Because the cumulative inflammatory burden is positively associated with cardiovascular events [29], the worsening cardiovascular profile resulting 
from chronic nicotine-induced HTN and mild RA should be of considerable concern, especially to smokers.

Chronic nicotine exposure during the onset of RA resulted in additional damage to the gastrocnemius muscles, despite heightened systemic inflammation and a reduction in IGF-1 caused by CIA-induced worsening of joint edema, thereby decreasing mobility [30]. The gastrocnemius muscle is composed mainly of type II muscle fibers, which support physical function and are the first to be recruited to prevent injury from a fall [31]. Thus, further loss of gastrocnemius muscle and premature mitochondrial dysfunction caused by chronic exposure to nicotine can predict earlier and worsened physical disability [32]. In contrast, muscle fiber size did not decrease further despite the greater muscle weight reduction caused by chronic nicotine exposure. In comparison, muscle fiber modification was observed beginning 12 weeks after exposure to cigarette smoke [33].

We also evaluated the effects of prophylactic administration of lavender and linalyl acetate on the properties of the $\mathrm{H}-\mathrm{CIA}$ model. Both lavender and linalyl acetate suppressed disease activity by reducing the level of IL-6. IL-6 has been shown to inhibit NF-KB activation, inducing excessive immunologic responses $[11,18]$. Lavender had limited effects on properties associated with systemic inflammation, such as paw edema, serum IL- 6 concentration and systolic BP. In addition to these anti-inflammatory effects, linalyl acetate prevented premature vascular endothelial damage and inhibited accelerated muscle atrophy caused by chronic nicotine exposure.

The effectiveness of linalyl acetate may result from its inhibition of inducible nitric oxide synthesis (NOS), which is enhanced by nicotine exposure [19]. IGF-1 enhances vascular NOS activity, inhibiting endothelial cell apoptosis [27]. Linalyl acetate-induced maintenance of IGF-1 levels promotes muscle anabolism and may contribute to the preservation of muscle mass. Moreover, linalyl acetate-associated increases in IGF1 are also encouraging, inasmuch as low serum IGF-1 has been found to predict early cardiovascular events in RA patients [34]. In addition, treatments to prevent or reverse muscle atrophy have focused on IGF-1-associated muscle anabolic pathways [35]. Vasodilation following linalyl acetate associated maintenance of vascular endothelium supports results showing that linalyl acetate preserves normotensive levels of diastolic and systolic BP [20]. Linalyl acetate has also been shown to regulate intracellular $\mathrm{Ca}^{2+}$ levels in vascular endothelial cells and smooth muscle cells, suggesting that the antihypertensive effects of linalyl acetate are associated with vasomotor enhancement [36]. Our finding, that linalyl acetate restores BP and endothelium thickness to near-normal levels is especially important, inasmuch as higher BP and atherosclerosis are risk factors for the development of cardiovascular diseases [27].

Although linalyl acetate did not completely prevent muscle loss, it prevented further loss of gastrocnemius muscle caused by chronic exposure to nicotine. Moreover, the ability of linalyl acetate to preserve total weight and IGF-1 levels suggests that linalyl acetate maintains overall physique, and that its ability to prevent muscle atrophy could be indirectly estimated. Lavender treatment tended to preserve muscle mass and fiber size even without significantly increasing IGF-1 levels, suggesting that the 
analgesic effect of lavender contributed to its maintenance of mobility [21,37]. The loss of muscle mass and function in our rat model of RA and HTN suggests that current pharmaceutical interventions have severe limitations due to their inability to reverse muscle weakness, because increased muscle mass does not guarantee improved muscle function [38]. Exercise is the only effective strategy to alleviate muscle weakness, while restoring muscle mass and mitochondrial homeostasis [39]. However, the ability of patients with physical disability to exercise is limited. Linalyl acetate may therefore be a novel therapeutic agent for preserving both muscle mass and function.

\section{Conclusions}

The present findings suggest that individuals with an environmental or genetic risk of RA should quit smoking, not only to reduce the risks of RA and HTN, but also to reduce the risks of cardiovascular disease and physical dysfunction associated with RA accompanied by HTN. Although lavender oil, which contains linalyl acetate as a main component, is easy to apply, it has a limited effect on some of the factors primarily involved in inhibiting inflammation. Prophylactic application of linalyl acetate in patients at risk for RA and tobacco-associated HTN may reduce the risk of cardiovascular diseases and preserve muscle function and even muscle mass.

\section{Abbreviations}

$\mathrm{BP}$

Blood pressure; ELISA:Enzyme-linked immunosorbent assay; HTN:Hypertension; H\&E:Hematoxylin and eosin; IGF-1:Insulin-like growth factor-1; IL-6:Interleukin-6; NOS:Nitric oxide synthesis; RA:Rheumatoid arthritis.

\section{Declarations}

\section{Acknowledgements}

Not applicable.

\section{Authors' contributions}

ES and GHS designed the study. ES, YSH, JML, YKS and GHS performed the study and wrote the manuscript. ES, YKS and GHS revised the manuscript. All authors read and approved the final manuscript.

\section{Funding}

This work was supported by a grant from the Basic Science Research Program through the National Research Foundation of Korea (NRF-2018R1D1A1B07050048) and a Korea University Grant.

\section{Availability of data and materials}


The data that support the findings of this study are available from the corresponding author on reasonable request.

\section{Ethics approval and consent to participate}

The animal experiments were approved by the institutional animal care and research committee of Korea University.

\section{Consent for publication}

Not applicable.

\section{Competing interest}

The authors declare that they have no competing interests.

\section{References}

1. Bremander A, Malm K, Andersson ML. Physical activity in established rheumatoid arthritis and variables associated with maintenance of physical activity over a seven-year period - a longitudinal observational study. BMC Rheumatol. 2020;4:53.

2. Bai T, Fang F, Li F, Ren Y, Hu J, Cao J. Sarcopenia is associated with hypertension in older adults: a systematic review and meta-analysis. BMC Geriatr. 2020;20:279.

3. Bajraktari IH, Rexhepi S, Berisha I, Lahu A, Kryeziu A, Durmishi B, et al. Prevalence of Asymptomatic Arterial Hypertension and Its Correlation with Inflammatory Activity in Early Rheumatoid Arthritis. Open Access Maced J Med Sci. 2017;5:641-4.

4. Molina E, del Rincon I, Restrepo JF, Battafarano DF, Escalante A. Mortality in Rheumatoid Arthritis (RA): factors associated with recording RA on death certificates. BMC Musculoskelet Disord. 2015;16:277.

5. van Breukelen-van der Stoep DF, van Zeben D, Klop B, van de Geijn GJ, Janssen HJ, van der Meulen $\mathrm{N}$, et al. Marked underdiagnosis and undertreatment of hypertension and hypercholesterolaemia in rheumatoid arthritis. Rheumatology. 2016;55:1210-6.

6. Pantell MS, Prather AA, Downing JM, Gordon NP, Adler NE. Association of Social and Behavioral Risk Factors With Earlier Onset of Adult Hypertension and Diabetes. JAMA Netw Open. 2019;2:e193933.

7. Gwinnutt JM, Verstappen SM, Humphreys JH. The impact of lifestyle behaviours, physical activity and smoking on morbidity and mortality in patients with rheumatoid arthritis. Best Pract Res Clin Rheumatol. 2020:101562.

8. Lesage-Meessen L, Bou M, Sigoillot JC, Faulds CB, Lomascolo A. Essential oils and distilled straws of lavender and lavandin: a review of current use and potential application in white biotechnology. Appl Microbiol Biotechnol. 2015;99:3375-85. 
9. Sanna MD, Les F, Lopez V, Galeotti N. Lavender (Lavandula angustifolia Mill.) Essential Oil Alleviates Neuropathic Pain in Mice With Spared Nerve Injury. Front Pharmacol. 2019;10:472.

10. Cardia GFE, Silva-Filho SE, Silva EL, Uchida NS, Cavalcante HAO, Cassarotti LL, et al. Effect of Lavender (Lavandula angustifolia) Essential Oil on Acute Inflammatory Response. Evid Based Complement Alternat Med. 2018;2018:1413940.

11. Aoe M, Ueno-lio T, Shibakura M, Shinohata R, Usui S, Arao Y, et al. Lavender Essential Oil and Its Main Constituents Inhibit the Expression of TNF-a-induced Cell Adhesion Molecules in Endothelial Cells. Acta Med Okayama. 2017;71:493-503.

12. Rambod M, Nazarinia M, Raieskarimian $F$. The prevalence and predictors of herbal medicines usage among adult rheumatoid arthritis patients: A case-control study. Complement Ther Med. 2018;41:220-4.

13. Nasiri A, Mahmodi MA. Aromatherapy massage with lavender essential oil and the prevention of disability in ADL in patients with osteoarthritis of the knee: A randomized controlled clinical trial. Complement Ther Clin Pract. 2018;30:116-21.

14. Sebai H, Selmi S, Rtibi K, Souli A, Gharbi N, Sakly M. Lavender (Lavandula stoechas L.) essential oils attenuate hyperglycemia and protect against oxidative stress in alloxan-induced diabetic rats. Lipids Health Dis. 2013;12:189.

15. Bojić M, Maleš Ž, Antolić A, Babić I, Tomičić M. Antithrombotic activity of flavonoids and polyphenols rich plant species. Acta Pharm. 2019;69:483-95.

16. Letizia CS, Cocchiara J, Lalko J, Api AM. Fragrance material review on linalyl acetate. Food Chem Toxicol. 2003;41:965-76.

17. Moon PD, Han NR, Lee JS, Kim HM, Jeong HJ. Effects of Linalyl Acetate on Thymic Stromal Lymphopoietin Production in Mast Cells. Molecules. 2018;23:1711.

18. Hsieh YS, Shin YK, Han AY, Kwon S, Seol GH. Linalyl acetate prevents three related factors of vascular damage in COPD-like and hypertensive rats. Life Sci. 2019;232:116608.

19. Kim JR, Kang P, Lee HS, Kim KY, Seol GH. Cardiovascular effects of linalyl acetate in acute nicotine exposure. Environ Health Prev Med. 2017;22:42.

20. Shin YK, Hsieh YS, Kwon S, Lee HS, Seol GH. Linalyl acetate restores endothelial dysfunction and hemodynamic alterations in diabetic rats exposed to chronic immobilization stress. J Appl Physiol (1985). 2018;124:1274-83.

21. Yu SH, Seol GH. Lavandula angustifolia Mill. Oil and Its Active Constituent Linalyl Acetate Alleviate Pain and Urinary Residual Sense after Colorectal Cancer Surgery: A Randomised Controlled Trial. Evid Based Complement Alternat Med. 2017;2017:3954181.

22. Moon HK, Kang P, Lee HS, Min SS, Seol GH. Effects of 1,8-cineole on hypertension induced by chronic exposure to nicotine in rats. J Pharm Pharmacol. 2014;66:688-93.

23. Alabarse PVG, Lora PS, Silva JMS, Santo RCE, Freitas EC, de Oliveira MS, et al. Collagen-induced arthritis as an animal model of rheumatoid cachexia. J Cachexia Sarcopenia Muscle. 2018;9:60312. 
24. Filippin LI, Teixeira VN, Viacava PR, Lora PS, Xavier LL, Xavier RM. Temporal development of muscle atrophy in murine model of arthritis is related to disease severity. J Cachexia Sarcopenia Muscle. 2013;4:231-8.

25. Oyenihi AB, Ollewagen T, Myburgh KH, Powrie YSL, Smith C. Redox Status and Muscle Pathology in Rheumatoid Arthritis: Insights from Various Rat Hindlimb Muscles. Oxid Med Cell Longev. 2019;2019:2484678.

26. Anyfanti P, Gavriilaki E, Douma S, Gkaliagkousi E. Endothelial Dysfunction in Patients with Rheumatoid Arthritis: the Role of Hypertension. Curr Hypertens Rep. 2020;22:56.

27. Conti E, Carrozza C, Capoluongo E, Volpe M, Crea F, Zuppi C, et al. Insulin-like growth factor-1 as a vascular protective factor. Circulation. 2004;110:2260-5.

28. Yang X, Chang Y, Wei W. Endothelial Dysfunction and Inflammation: Immunity in Rheumatoid Arthritis. Mediators Inflamm. 2016;2016:6813016.

29. Karpouzas GA, Ormseth SR, Hernandez E, Budoff MJ. Impact of Cumulative Inflammation, Cardiac Risk Factors, and Medication Exposure on Coronary Atherosclerosis Progression in Rheumatoid Arthritis. Arthritis Rheumatol. 2020;72:400-8.

30. de Oliveira Nunes Teixeira V, Filippin LI, Viacava PR, de Oliveira PG, Xavier RM. Muscle wasting in collagen-induced arthritis and disuse atrophy. Exp Biol Med (Maywood). 2013;238:1421-30.

31. Wang E, Nyberg SK, Hoff J, Zhao J, Leivseth G, Tørhaug T, et al. Impact of maximal strength training on work efficiency and muscle fiber type in the elderly: Implications for physical function and fall prevention. Exp Gerontol. 2017;91:64-71.

32. Jagpal A, Navarro-Millán I. Cardiovascular co-morbidity in patients with rheumatoid arthritis: a narrative review of risk factors, cardiovascular risk assessment and treatment. BMC Rheumatol. 2018;2:10.

33. Zhang Y, Gao J, Luo Y. The effect of various durations of cigarette smoke exposure on muscle fibre remodeling in rat diaphragms. Biomed Pharmacother. 2019;117:109053.

34. Yang J, Yuan Y, Hu X, Han R, Chen M, Wang M, et al. Low serum levels of insulin-like growth factor-1 are associated with an increased risk of rheumatoid arthritis: a systematic review and meta-analysis. Nutr Res. 2019;69:9-19.

35. Lynch GS. Update on emerging drugs for sarcopenia - age-related muscle wasting. Expert Opin Emerg Drugs. 2008;13:655-73.

36. You JH, Kang P, Min SS, Seol GH. Bergamot essential oil differentially modulates intracellular Ca2 + levels in vascular endothelial and smooth muscle cells: a new finding seen with fura-2. J Cardiovasc Pharmacol. 2013;61:324-8.

37. Mochizuki T, Ikari K, Yano K, Okazaki K. Evaluation of factors associated with locomotive syndrome in Japanese elderly and younger patients with rheumatoid arthritis. Mod Rheumatol. 2019;29:733-6.

38. Wagner KR, Fleckenstein JL, Amato AA, Barohn RJ, Bushby K, Escolar DM, et al. A phase I/Iltrial of MYO-029 in adult subjects with muscular dystrophy. Ann Neurol. 2008;63:561-71. 
39. Lo JH, U KP, Yiu T, Ong MT, Lee WY. Sarcopenia: Current treatments and new regenerative therapeutic approaches. J Orthop Translat. 2020;23:38-52.

\section{Figures}

Fig. 1

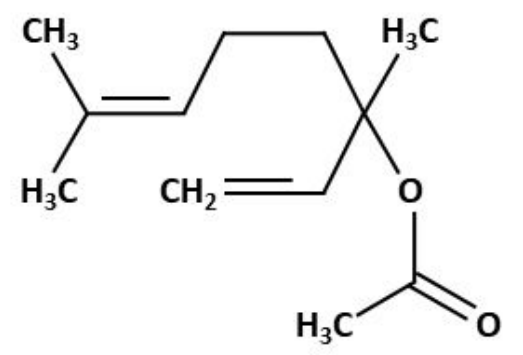

Figure 1

Structural formula of linalyl acetate 
Fig. 1

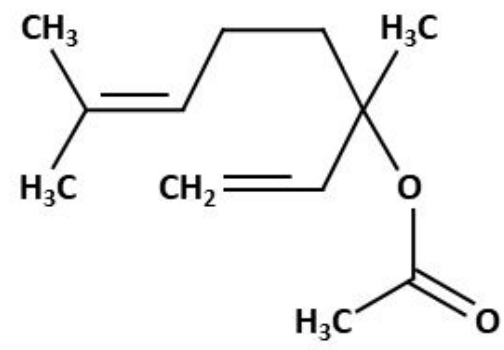

Figure 1

Structural formula of linalyl acetate 
Fig. 2

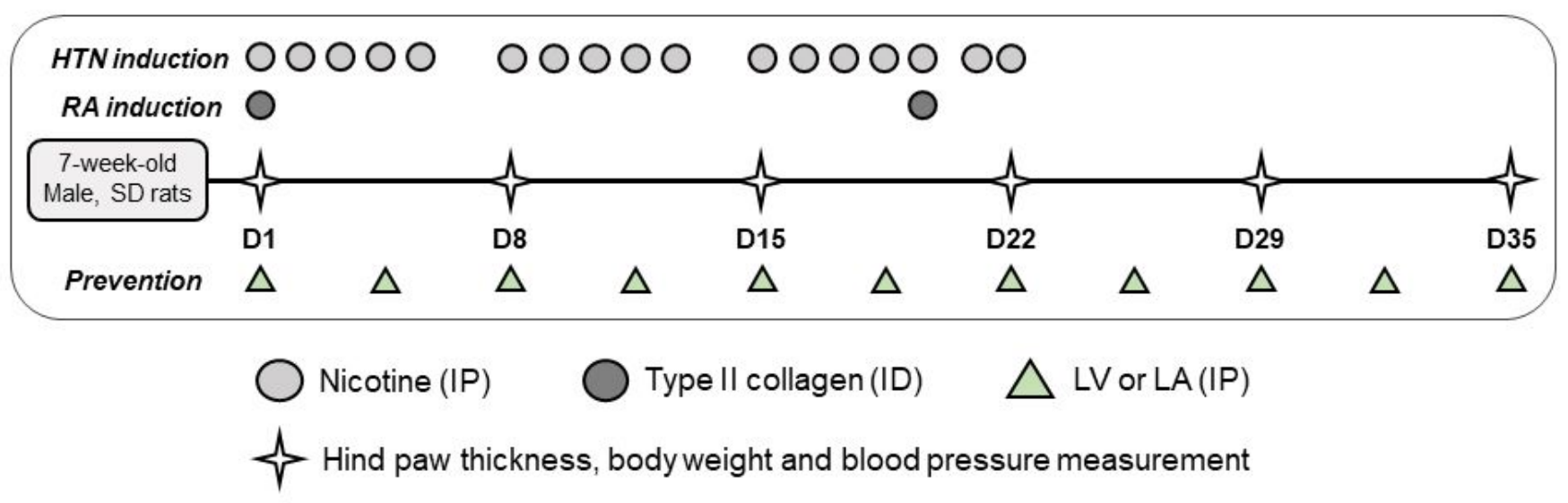

Figure 2

Schematic diagram of the experimental design 
Fig. 2

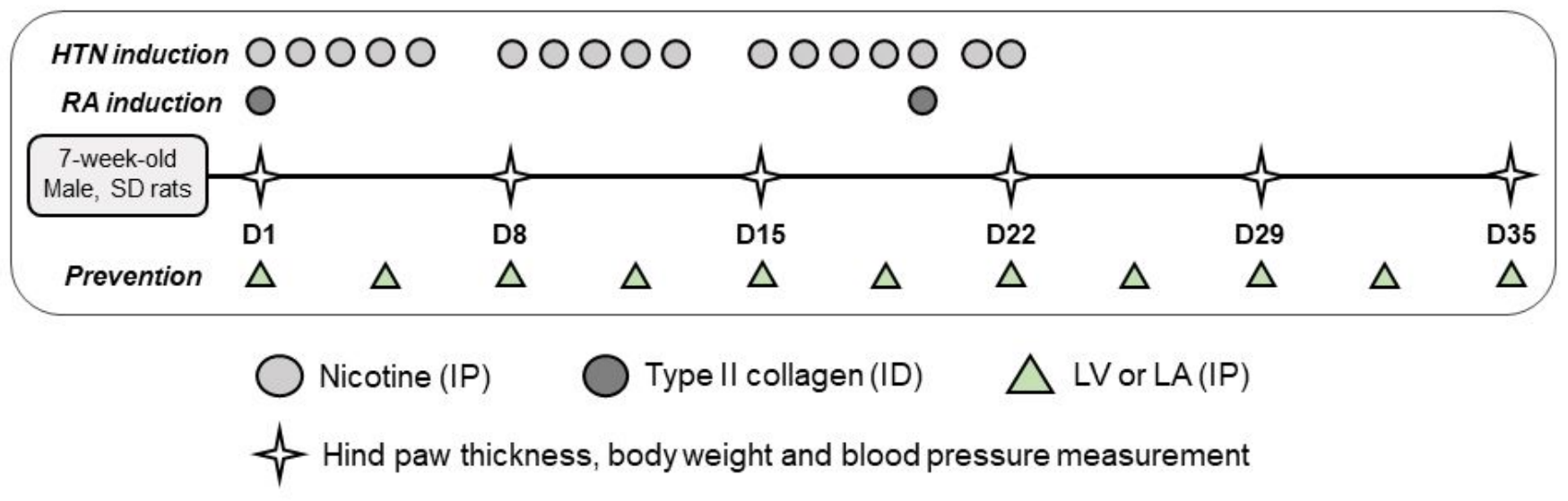

Figure 2

Schematic diagram of the experimental design 
Fig. 3
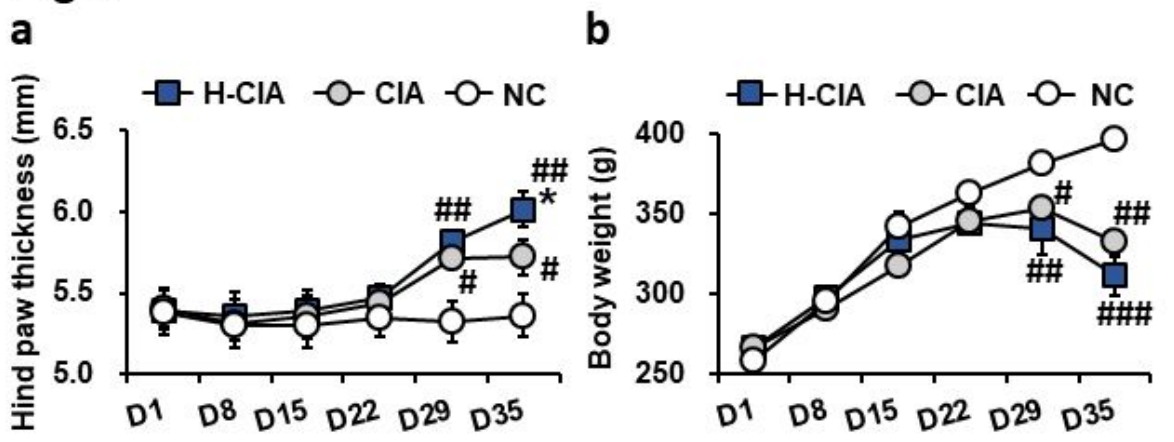

C

d

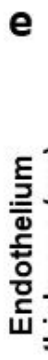
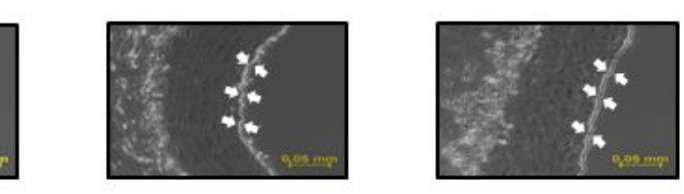

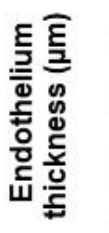
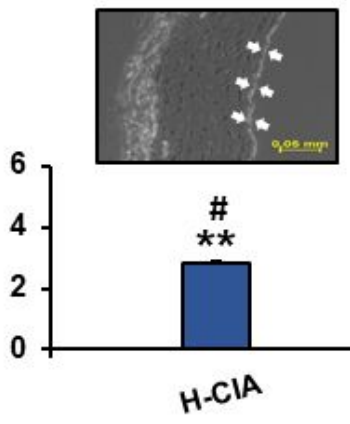

h

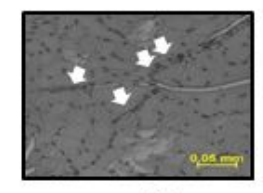

H.CIA

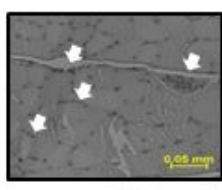

CIA

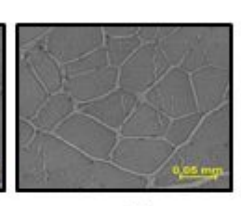

NC

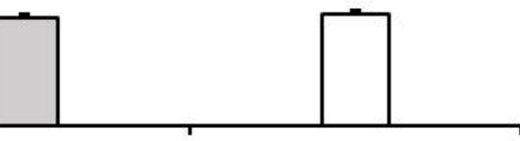

NC

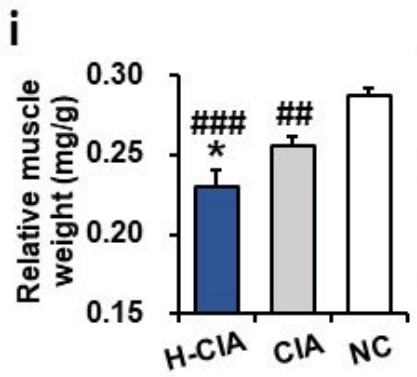

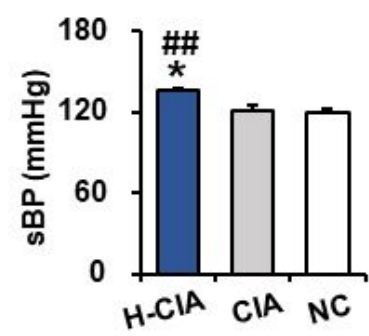

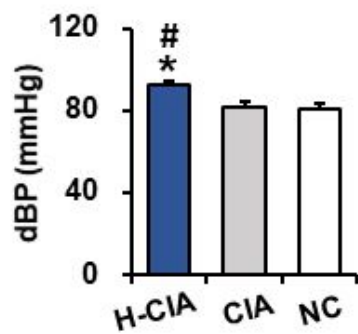

f

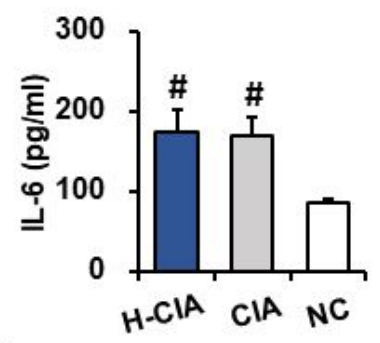

k

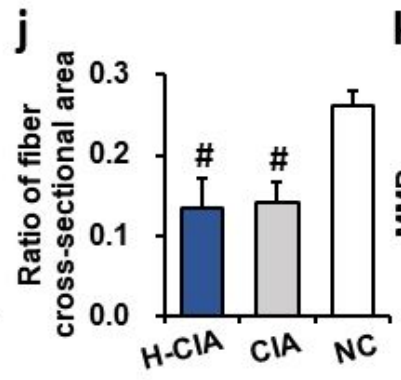

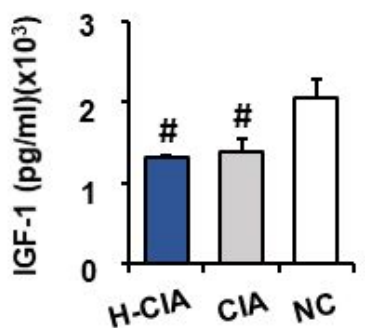

g

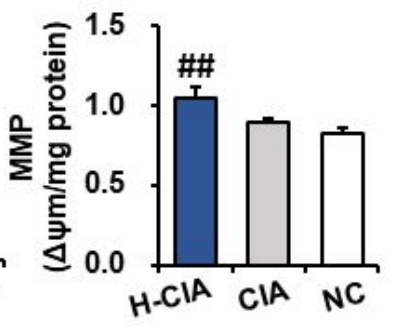

Figure 3

Effects of co-occurrence of chronic nicotine-induced hypertension and collagen-induced arthritis. a Hind paw thickness and b body weight during the experimental period. c Systolic and d diastolic blood pressure (BP) on the last day of the experiment. e Endothelium thickness of the thoracic aorta with representative hematoxylin and eosin (H\&E) images. Serum concentrations of $f$ interleukin-6 (IL-6) and g insulin-like growth factor-1 (IGF-1). h H\&E-stained images of gastrocnemius muscle, showing morphological differences. i Ratio of wet weight of gastrocnemius muscle to total body weight and $\mathrm{j}$ ratio of muscle fiber cross-sectional area. $\mathrm{k}$ Mitochondrial membrane potential (MMP) of the gastrocnemius muscle. All values are shown as mean $\pm \operatorname{SEM}(n=6 \sim 8)$. \# $p<0.05, \# \# p<0.01$, \#\#\#p<0.001 compared with the normal control (NC) group; * $p<0.05$, $* \star p<0.01$, compared with the collagen induced arthritis (CIA) group. H-ClA: CIA accompanied by chronic nicotine-induced hypertension 
Fig. 3
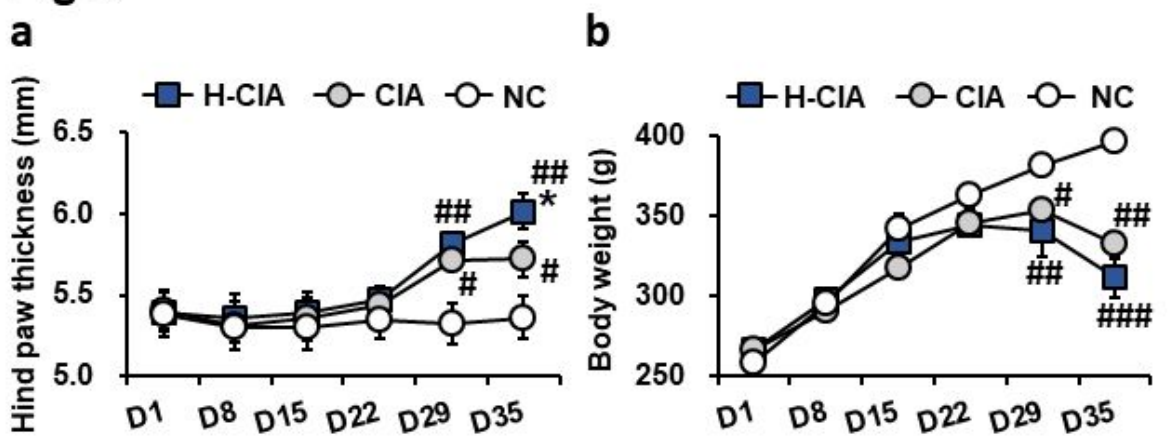

C

d

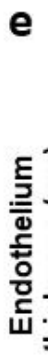
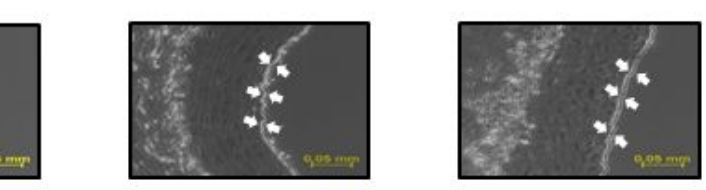

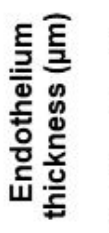
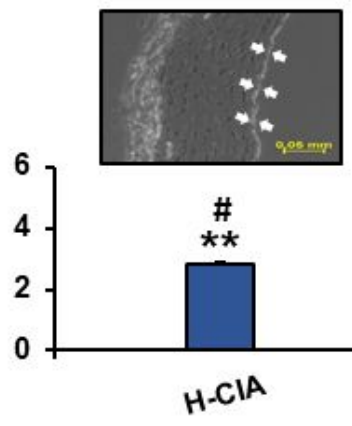

h

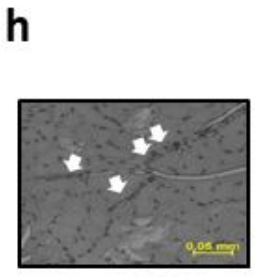

H-CIA

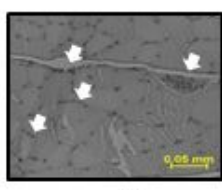

CIA

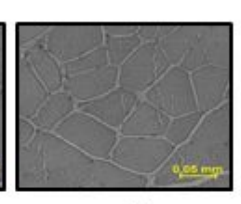

NC

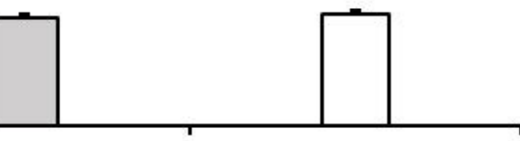

NC

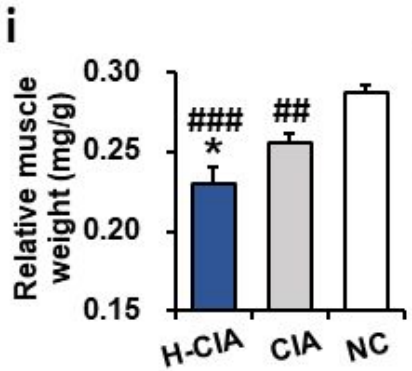

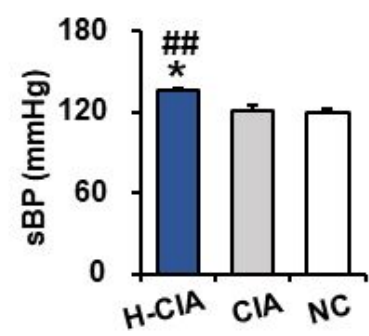

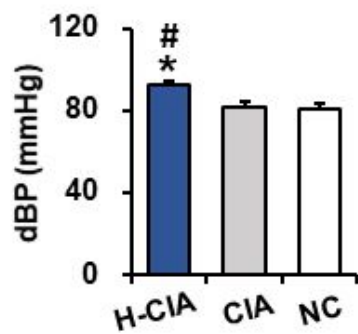

g
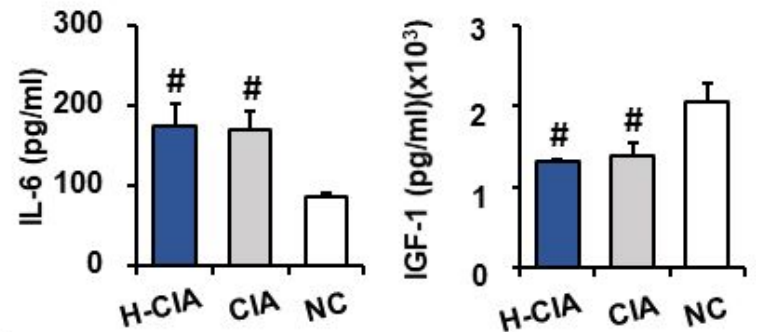

k
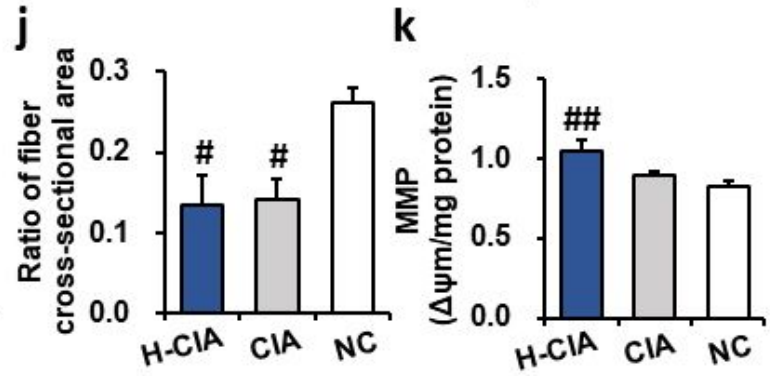

Figure 3

Effects of co-occurrence of chronic nicotine-induced hypertension and collagen-induced arthritis. a Hind paw thickness and b body weight during the experimental period. c Systolic and d diastolic blood pressure (BP) on the last day of the experiment. e Endothelium thickness of the thoracic aorta with representative hematoxylin and eosin (H\&E) images. Serum concentrations of $f$ interleukin-6 (IL-6) and g insulin-like growth factor-1 (IGF-1). h H\&E-stained images of gastrocnemius muscle, showing morphological differences. i Ratio of wet weight of gastrocnemius muscle to total body weight and $\mathrm{j}$ ratio of muscle fiber cross-sectional area. $\mathrm{k}$ Mitochondrial membrane potential (MMP) of the gastrocnemius muscle. All values are shown as mean $\pm \operatorname{SEM}(n=6 \sim 8)$. \# $p<0.05, \# \# p<0.01$, \#\#\#p<0.001 compared with the normal control (NC) group; * $p<0.05$, $* \star p<0.01$, compared with the collagen induced arthritis (CIA) group. H-ClA: CIA accompanied by chronic nicotine-induced hypertension 
Fig. 4
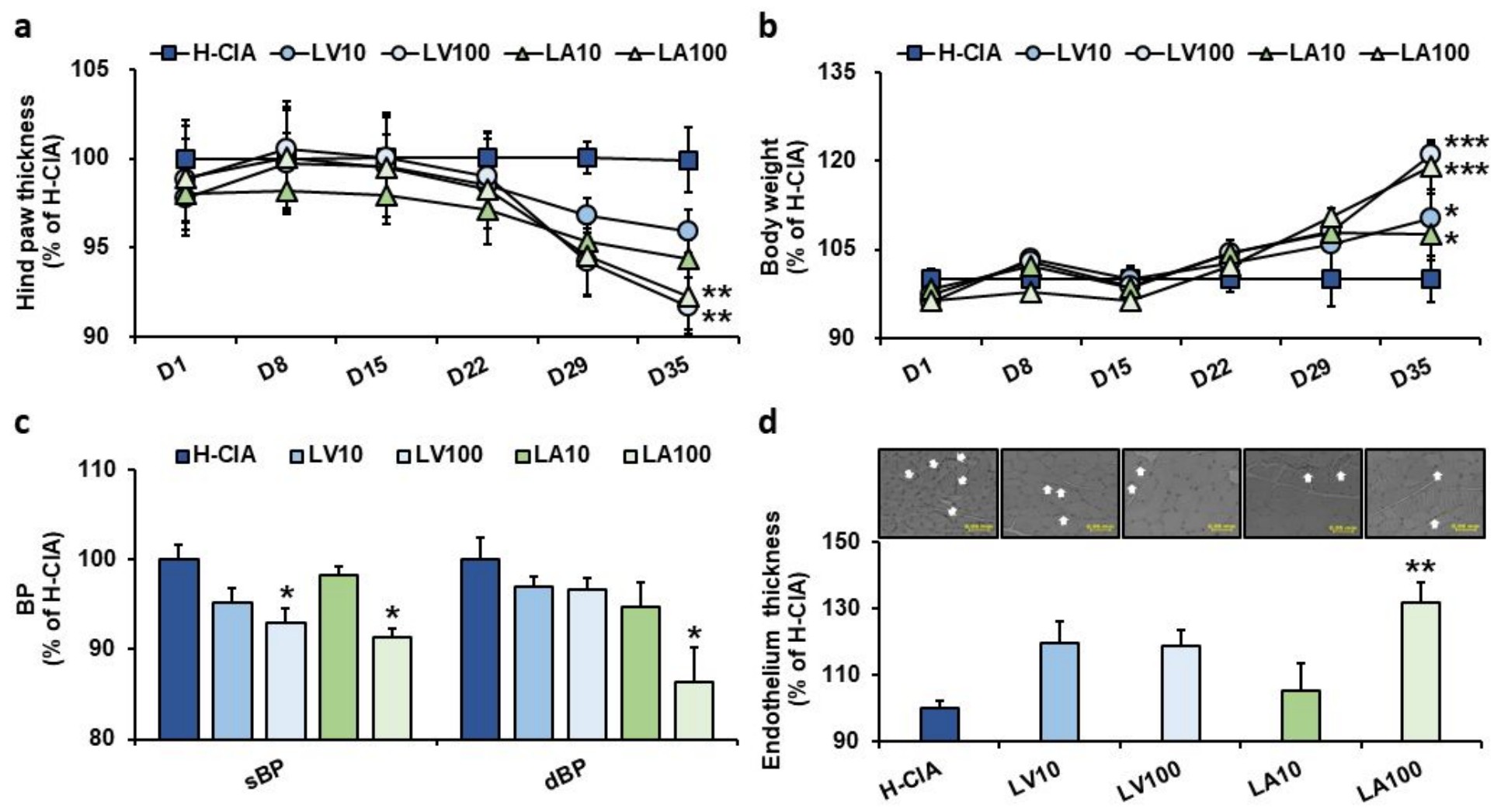

Figure 4

Effects of prophylactic application of lavender and linalyl acetate on clinical observations and vascular homeostasis. a Hind paw thickness, b body weight, and c blood pressure (BP) measured once a week during the experimental period. $d$ Endothelium thickness of the thoracic aorta with representative hematoxylin and eosin (H\&E) images. All values were shown as mean $\pm \operatorname{SEM}(n=6 \sim 8)$. ${ }^{*}<0.05$, ** $p<$ 0.01 compared with the $\mathrm{H}$-CIA group. $\mathrm{H}-\mathrm{CIA}$, collagen induced arthritis accompanied by chronic nicotineinduced hypertension; LV10: lavender 10 mg/kg; LV100: lavender 100 mg/kg; LA10: linalyl acetate 10 $\mathrm{mg} / \mathrm{kg}$; LA100: linalyl acetate $100 \mathrm{mg} / \mathrm{kg}$ 
Fig. 4
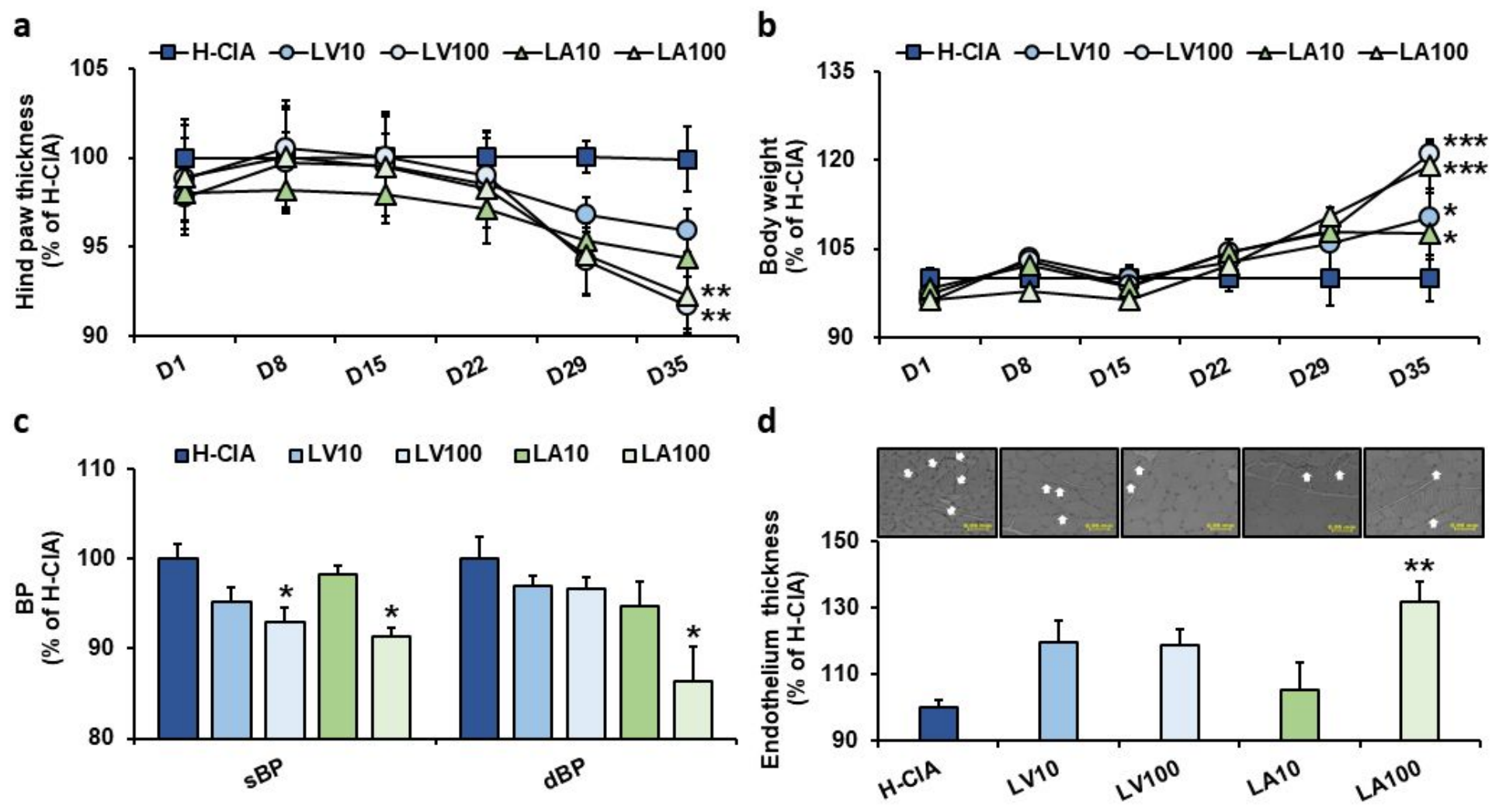

Figure 4

Effects of prophylactic application of lavender and linalyl acetate on clinical observations and vascular homeostasis. a Hind paw thickness, b body weight, and c blood pressure (BP) measured once a week during the experimental period. $d$ Endothelium thickness of the thoracic aorta with representative hematoxylin and eosin (H\&E) images. All values were shown as mean $\pm \operatorname{SEM}(n=6 \sim 8)$. ${ }^{*}<0.05$, ** $p<$ 0.01 compared with the $\mathrm{H}$-CIA group. $\mathrm{H}-\mathrm{CIA}$, collagen induced arthritis accompanied by chronic nicotineinduced hypertension; LV10: lavender 10 mg/kg; LV100: lavender 100 mg/kg; LA10: linalyl acetate 10 $\mathrm{mg} / \mathrm{kg}$; LA100: linalyl acetate $100 \mathrm{mg} / \mathrm{kg}$ 
Fig. 5

a

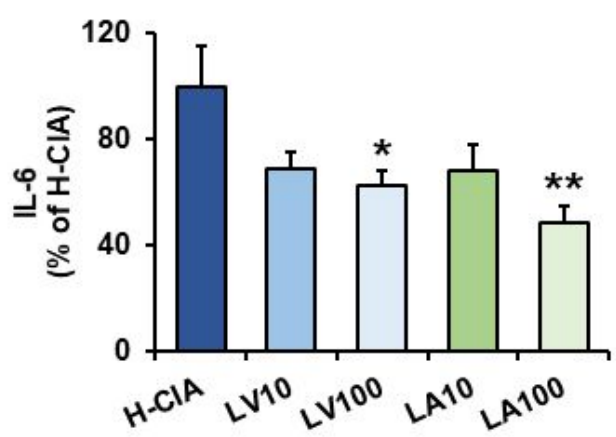

d

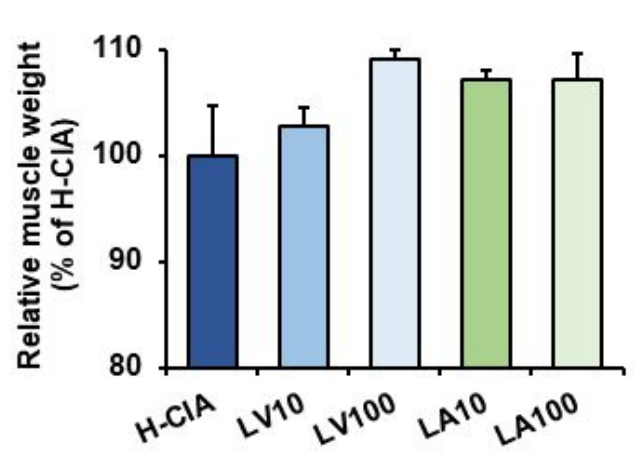

b

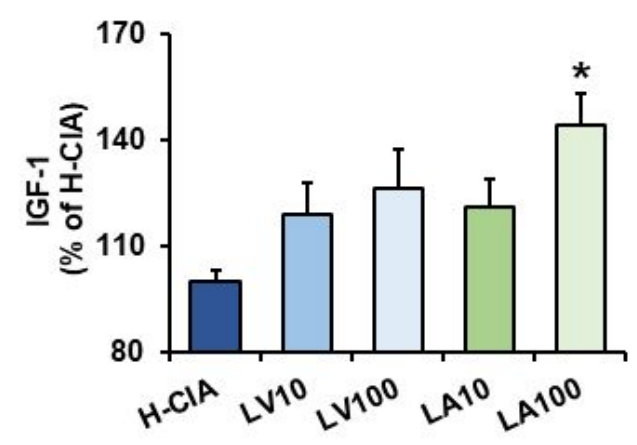

e

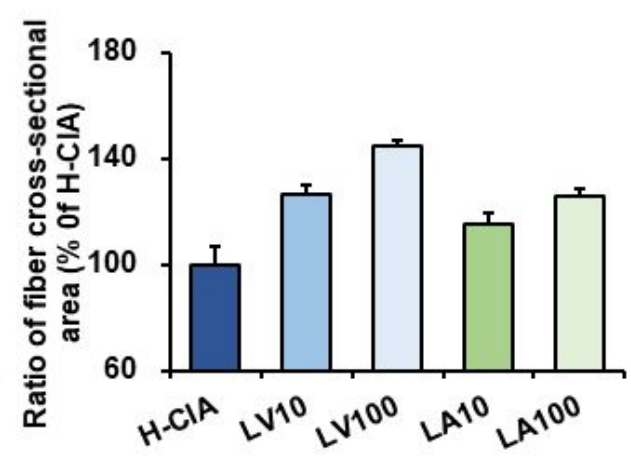

C

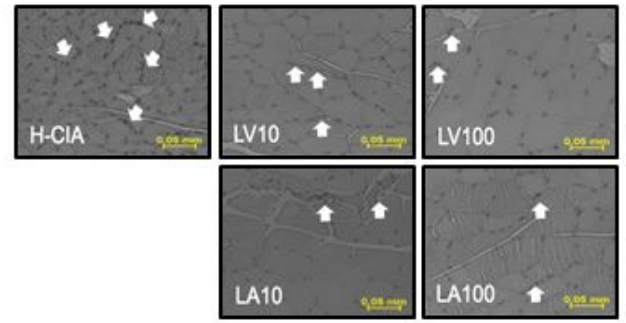

f

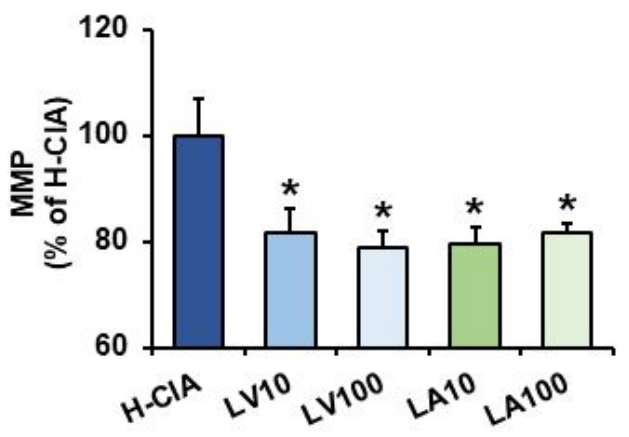

Figure 5

Effects of prophylactic application of lavender and linalyl acetate on serum and gastrocnemius muscle analysis. Serum concentration of a interleukin-6 (IL-6) and b insulin-like growth factor-1 (IGF-1). c hematoxylin and eosin (H\&E) images of gastrocnemius muscle showing morphological differences. $d$ Ratio of wet weight of gastrocnemius muscle to total body weight and e ratio of muscle fiber crosssectional area. $f$ Mitochondrial membrane potential (MMP) of the gastrocnemius muscle. Values are shown as mean \pm SEM $(n=6 \sim 8)$. ${ }^{*} p<0.05,{ }^{* *} p<0.01$ compared with the H-CIA group. H-CIA, collagen induced arthritis accompanied by chronic nicotine-induced hypertension; LV10: lavender $10 \mathrm{mg} / \mathrm{kg}$; LV100: lavender 100 mg/kg; LA10: linalyl acetate 10 mg/kg; LA100: linalyl acetate 100 mg/kg 
Fig. 5

a

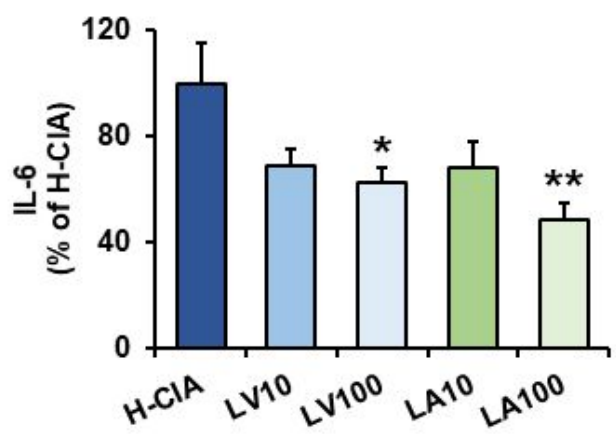

d

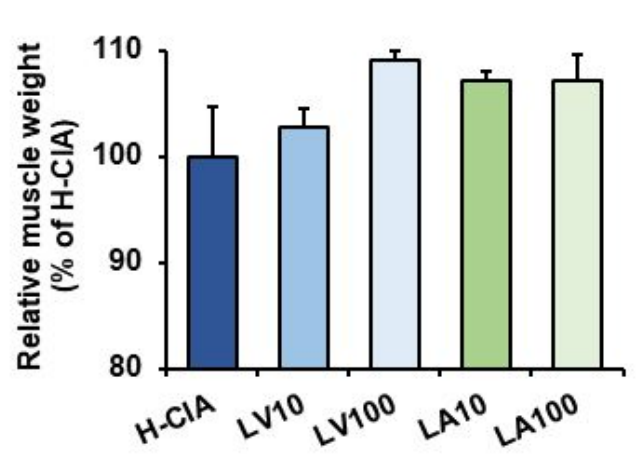

b

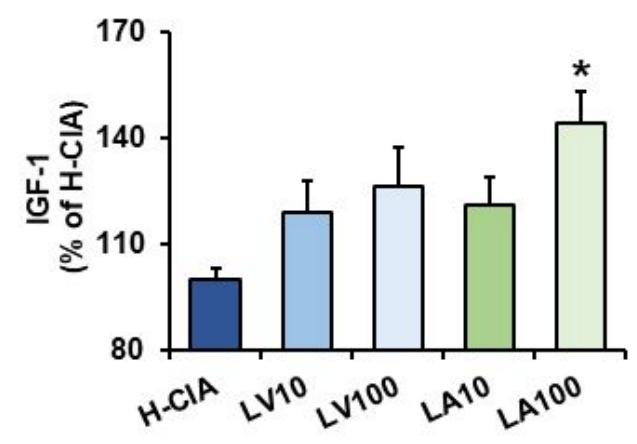

e

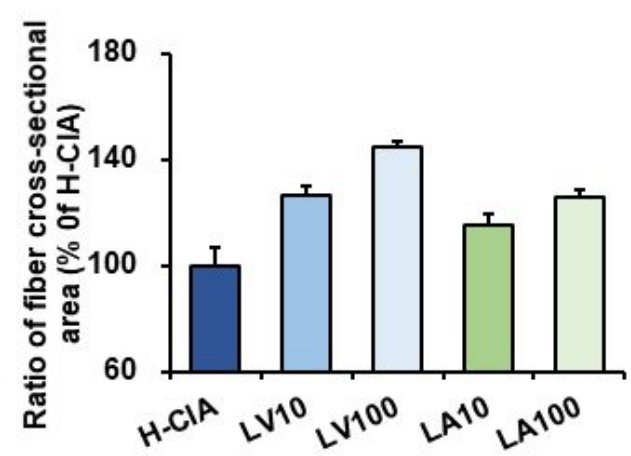

C

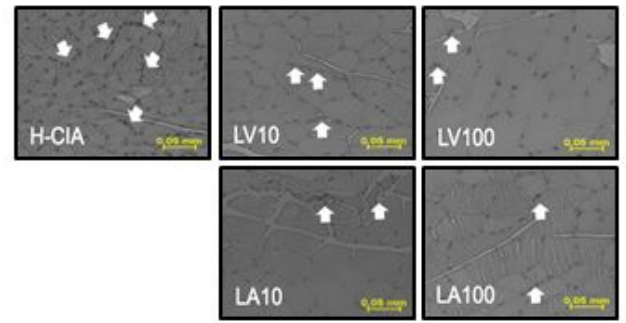

f

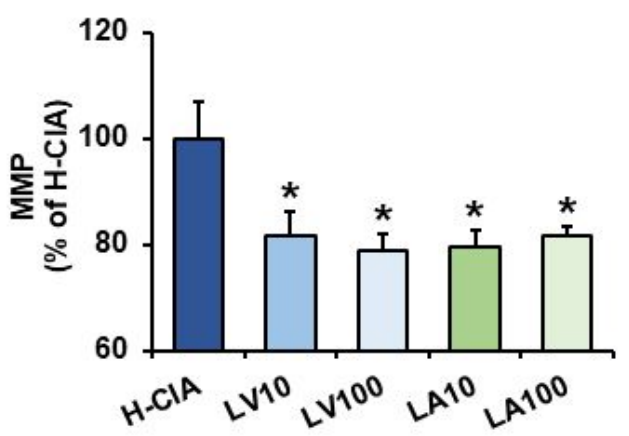

Figure 5

Effects of prophylactic application of lavender and linalyl acetate on serum and gastrocnemius muscle analysis. Serum concentration of a interleukin-6 (IL-6) and b insulin-like growth factor-1 (IGF-1). c hematoxylin and eosin (H\&E) images of gastrocnemius muscle showing morphological differences. $d$ Ratio of wet weight of gastrocnemius muscle to total body weight and e ratio of muscle fiber crosssectional area. $f$ Mitochondrial membrane potential (MMP) of the gastrocnemius muscle. Values are shown as mean \pm SEM $(n=6 \sim 8)$. ${ }^{*} p<0.05,{ }^{* *} p<0.01$ compared with the H-CIA group. H-CIA, collagen induced arthritis accompanied by chronic nicotine-induced hypertension; LV10: lavender $10 \mathrm{mg} / \mathrm{kg}$; LV100: lavender 100 mg/kg; LA10: linalyl acetate 10 mg/kg; LA100: linalyl acetate 100 mg/kg 